Z. Klin. Chem. Klin. Biochem.

13. Jg. 1975, S. 21-24

\title{
Eisen-Bestimmung mit dem CentrifiChem System
}

\author{
Von H.-G. Eisenwiener \\ Laboratorien der Abteilung Diagnostica, F. Hoffman-La Roche \& Co. Ch-4133 Schweizerhalle
}

(Eingegangen am 22. August/12. Oktober 1974)

\begin{abstract}
Die Bestimmung des Eisens wurde auf das CentrifiChem System adaptiert. Das an Transferrin gebundene Eisen wird durch ein Detergenz abgespalten, durch Natriumdithionit zu Fe ${ }^{++}$reduziert und mit Bathophenanthrolindisulfonat bestimmt. Die Durch führung besteht aus 2 Läufen für Analysen-Leerwert und Analyse. Obwohl die eigentliche Reaktionszeit äußerst kurz ist, wird eine Reaktionszeit von 8-10 min für Analysen-Leerwert und Analyse vorgeschlagen, da während der Zentrifugation und unter dem Einfluß des Teepols eine Klärung auftritt. Es werden $2 \times 100 \mu l$ Serum benötigt, etwa 80 Bestimmungen können pro Stunde durchgeführt werden.
\end{abstract}

\section{Determination of iron with CentrifiChem System}

The determination of iron was adapted to the CentrifiChem System. The iron bound to transferrin is freed with a detergent, reduced to $\mathrm{Fe}^{++}$with sodium dithionite, and determined with bathophenanthroline disulphonate. The operation consists of one run for the blank value and one analy tical run. Although the actual reaction time is extremely short, a reaction time of $8-10$ min is recommended for both the blank value and the analysis. This ensures adequate clearing during centrifugation under the influence of Teepol.

$2 \times 100 \mu l$ serum are required, and 80 determinations per hour are possible.

Ein Charakteristikum der Zentrifugalanalysatoren besteht darin, daß nur zu einem Zeitpunkt ein einziges Vielkomponenten-Reagenz durch Zentrifugalmischung mit der Probe zur Reaktion gelangt. Zu einem späteren Zeitpunkt, ev. nach Klärung des Probe-Reagenzgemisches oder nach einer Inkubationszeit ist es nicht möglich, ein weiteres Reagenz zum Reaktionsgemisch hinzuzufügen. Eine Enteiweißung ist ebenfalls nicht möglich. Aus diesen apparativen Gegebenheiten folgt, daß die Bestimmung des Eisens mit Zentrifugalanalysatoren nach einer Methode ohne Enteiweißung und mit einem einzigen Reagenz zu erfolgen hat:

\section{Methodik}

\section{Prinzip}

Die Abspaltung des Eisens vom Transferrin kann sowohl durch Salzsäure $(1,2,3)$ mit anschließender Enteiweißung als auch mit dem anionenaktiven Detergenz Teepol $(4,5,6)$ vorgenommen werden.

Leider ließ sich das von $K$. Lauber (4) entwickelte Pipettierschema ${ }^{1}$ ) nicht auf das CentrifiChem System übertragen, da es sich um ein Zweischritt-Pipettierschema handelt. Im 1. Schritt wird mit einem Reagenz, bestehend aus Acetatpuffer-Teepol und Natriumdithionit, das Eisen von Transferrin abgespalten und das $\mathrm{Fe}^{3+} \mathrm{zu} \mathrm{Fe}{ }^{2+}$ reduziert. Die Abspaltung des Eisens von Transferrin und dessen Reduktion gehen äußerst rasch vor sich, jedoch stellt sich bei den meisten Seren nicht sofort ein stabiler

1) Roche Diagnostica Test: Eisen, Art.Nr.1006
Extinktionswert ein, da durch das Teepol eine Klärung des Reaktionsgemisches auftritt. Bei lipämischen Seren ist eine Wartezeit bisweilen von 10-15 min notwendig. Wenn der Extinktionswert stabil ist, wird die Extinktion auf 0 gestellt, Bathophenanthrolindisulfonat-Lösung zugesetzt und die Extinktion gemessen. Da die Extinktion vor der Zugabe des Farbreagenz auf 0 gestellt wurde, bedeutet die erhaltene Extinktion nach Zugabe des Farbreagenz die Differenz aus Extinktion der Analyse und Analysen-Leerwert.

Der CentrifiChem Adaptation liegt ebenfalls eine Berücksichtigung des Analysen-Leerwertes zugrunde.

In einem Vorlauf wird Reagenz, bestehend aus Acetatpuffer, Teepol und Natriumdithionit, mit Probe gemischt und am Ausdruck beobachtet, bis der Analysen-Leerwert nicht mehr abnimmt infolge Klärung durch Teepol und Zentrifugation. Dieser Analysen-Leerwert stellt die Extinktion des Probe-ReagenzGemisches nach vorangehender Klärung dar. Die Extinktionen dieser Analysen-Leerwerte werden auf Kanal I gespeichert.

In dem darauffolgenden 2. Lauf wird dem gleichen Reagenz zusätzlich Bathophenanthrolindisulfonsäure zugesetzt und als Reaktionszeit diejenige gewählt, nach welcher sich beim Analysen-Leerwert die Extinktion nicht mehr änderte. Die Messung erfolgt gegen Reagenz + Wasser. Die gemessenen Extinktionen des 2. Laufs werden auf Kanal II gespeichert.

Der erhạttene Ausdruck stellt die Differenz zwischen Kanal II und I dar.

$E=E(A) \rightarrow R L-E(A L) \rightarrow \mathrm{H}_{2} \mathrm{O}$

$\mathrm{E}=$ erhaltener Extinktionswert im Ausdruck

$\mathbf{E}(\mathbf{A}) \rightarrow \mathbf{R L}=$ gemessene Extinktion der Analyse gegen Reagenzien-Leerwert im 2. Lauf; Werte sind auf Kanal II gespeichert.

$E(R L) \rightarrow \mathrm{H}_{2} \mathrm{O}=$ gemessene Extinktion des Analysen-Leerwertes gegen Wasser nach „Klärung“" im 1. Lauf; Werte auf Kanal I gespeichert. 


\section{Reagenzicn}

A : Teepol in Acetatpuffer, $\mathrm{pH}=5,8(40 \%)$

B : Natriumdithionit p.a.

C : Bathophenanthrolindisulfonat $7,5 \mathrm{mmol} / 1$

Die Lösungen $A$ und $C$ sowie das Natriumdithionit sind auch im Roche Diagnostica Test-Kit Eisen enthalten.

Gebrauchsfertiges Reagenz für Analysen-Leerwert:

$\mathrm{Zu} 10 \mathrm{ml}$ Lösung A werden etwa $200 \mathrm{mg}$ Natriumdithionit gegeben.

Gebrauchsfertiges Reagenz für Analyse:

$\mathrm{Zu} 10 \mathrm{ml}$ Lösung A werden etwa $200 \mathrm{mg}$ Natriumdithionit und $250 \mu$ Bathophenanthrolindisulfonat-Lösung gegeben.

\section{Vorgehen}

Zwischen der Empfindlichkeit, Menge an einzusetzender Probe, dem Carry over und dem Signal-Rauschverhältnis mußte ein Kompromiß eingegangen werden. Da die Empfindlichkeit aller Eisenbestimmungsmethoden nicht besonders groß ist, wird pro manueller Bestimmung je nach Testvorgehen 500-2000 $\mu$ l Serum eingesetzt. Das auf das CentrifiChem System adaptierte Verfahren kommt für Analysen-Leerwert und Analyse mit je $100 \mu \mathrm{l}$ Probenmaterial aus. Da der Carry over vom Diluentvolumen abhängt und das Diluentvolumen einmal mengenmäßig durch den Pipettor begrenzt ist und zum anderen durch Erhöhung des Endvolumens die gemessenen Extinktionsdifferenzen verkleinert, wurde das Probenvolumen manuell in die Bohrung für die Probe pipettiert. Das jeweilige Reagenz wurde mit dem Pipettor unter den Einstellungen: SAMPLE : 00; SAMPLE + DILUENT : 00; Reagenzvolumen: $250 \mu$ l pipettiert.

Die Meßwellenlänge war $550 \mathrm{~nm}$, die zur Aufklärung benötigte Zeit (=Reaktionszeit) lag zwischen'5 und 12 Minuten. Im allgemeinen ist eine Reaktionszeit von 9 Minuten ausreichend.

Die Berechnung erfolgte mit einem Standard von $17,9 \mu \mathrm{mol} / 1$ $(1,00 \mathrm{mg} / 1)$.

\section{Ergebnisse}

Das adaptierte Verfahren wurde hinsichtlich Linearität, Richtigkeit, Recovery und Präzision sowohl in der Serie, von Lauf zu Lauf als auch von Tag zu Tag überprüft. Weiterhin wurden Paralleluntersuchungen mit der manuellen Methode durchgefuhrt und die Regressionsgerade und Korrelation berechnet.

\section{Linearität}

Der Linearitätsbereich wurde bis $179 \mu \mathrm{mol} / 1$ Eisen $(10,00 \mathrm{mg} / 1)$ überprüft. Aus Abbildung 1 geht ein gut linearer Zusammenhang zwischen Meßwert (relative Extinktionsdifferenz bei $550 \mathrm{~nm}$, Filterfaktoreinstellung 500) hervor. Der bezeichnete Konzentrationsfaktor wäre 248,6 ( $\mu \mathrm{mol} / \mathrm{l})$ bzw. $13889(\mathrm{mg} / \mathrm{l})$. Ein Rauschpegel von 0,001 bis 0,002 Extinktionseinheiten würde demnach einem Fehler von etwa $0,4 \mu \mathrm{mol} / 1(20 \mu \mathrm{g} / \mathrm{l})$ entsprechen.

\section{Richtigkeit}

Die Richtigkeit wurde mit Hilfe von Kontrollseren humanen und tierischen Ursprungs überprüft (Tab. 1). Je nach Berechnung mit wäßrigem Standard, Kontrollserum von Dade, von Hyland oder Serionorm wurden

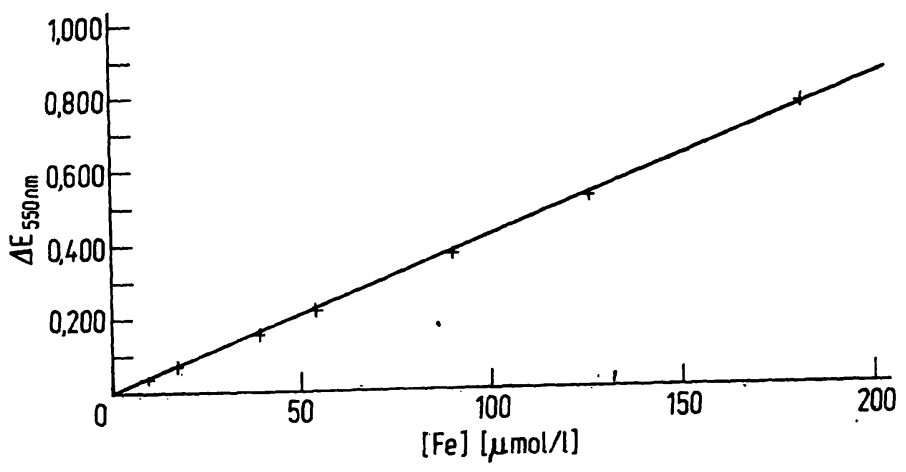

Abb. 1. Überprüfung des Linearitätsbereiches; relative Extinktionsdifferenz bei $550 \mathrm{~nm}$ aufgetragen gegen Konzentration in $\mu \mathrm{mol} / \mathrm{l}$.

etwas unterschiedliche Resultate erhalten. Die gefundenen Ergebnisse lagen jedoch im allgemeinen im jeweils angegebenen Deklarationsbereich. Bei den Seren Monitrol I und II wurden Werte an der oberen Grenze erhalten. Bei diesen beiden Seren wäre eine etwạs längere Reaktionszeit infolge Trübung erforderlich gewesen. Diè manuell nach der gleichen Methode ermittelten Werte lagen bei 700 (Monitrol I) bzw. 1680 (Monitrol II) $\mu \mathrm{g} / 1$ Eisen. Jedoch wurde das adaptierte Verfahren einheitlich für alle Proben mit einer Reaktionszeit von $10 \mathrm{~min}$ angewendet.

\section{Recovery-Untersuchungen}

Die Recovery-Untersuchungen mußten so geführt werden, daß sie eine Aussage lieferten sowohl für zugesetzte $\mathrm{Fe}^{3+}$-Ionen als auch für die Abspaltung des Eisens vom Transferrin. Deshalb wurden sowohl lyophilisierte Kontrollseren in wäßrigen Lösungen von $\mathrm{Fe}^{3+}$ als auch in Seren bzw. Kontrollseren aufgelôsțt.

\section{Recovery der Bestimmung von zugesetztem $\mathrm{Fe}^{+++}$}

Diese Recovery-Untersuchungen wurden so durchgeführt, daß Kontrollseren sowohl in dest. Wasser als auch in wäßrigen Verdünnungsreihen von $\mathrm{Fe}^{3+}$ aufgelöst wurden. Es wurde bis zu $35,8 \mu \mathrm{mol} / 1 \mathrm{Fe}^{+++}(2,00 \mathrm{mg} / \mathrm{l})$ zugesetzt. Der im Kontrollserum gefundene Wert wurde als Ausgangsbasis zur Berechnung der Recovery genommen. Der Mittelwert aus 6 Recovery-Untersuchungen lag bei $99 \%$.

\section{Recovery der Bestimmung unter Einschluß der Abspal- tung von Transferrin}

Diese Recovery-Untersuchungen wurden so durchgefuhrt, daß lyophilisierte Kontrollseren in Kontrollseren aufgelöst und Verdünnungsreihen angesetzt wurden. Der Mittelwert aus 6 Recovery-Untersuchungen lag bei $98 \%$.

\section{Präzision}

Bei Zentrifugalanalysatoren ist zu unteršcheiden zwischen Präzision im Lauf, von Lauf zu Lauf und von Tag zu 
Tab. 1. Prüfung auf Richtigkeit der Methode mit verschiedenen Kontrollseren.

\begin{tabular}{|c|c|c|c|}
\hline Kontrollserum & Methoden & $\begin{array}{l}\text { Deklaration } \\
{[\mu \mathrm{g} / \mathrm{l}]}\end{array}$ & $\begin{array}{l}\text { gefundener Wert } \\
{[\mu \mathrm{g} / 1]}\end{array}$ \\
\hline $\begin{array}{l}\text { Hyland I } \\
0369 \text { G004Al }\end{array}$ & Henry et al., Ferro-Check II, Hyland & $750-850-950$ & 810 \\
\hline $\begin{array}{l}\text { Hyland II } \\
\text { O368 G002Al }\end{array}$ & Henry et al., Ferro-Check II, Hyland & $1870-2050-2230$ & 1950 \\
\hline $\begin{array}{l}\text { Moni-Trol I } \\
125 \mathrm{~A}, \mathrm{~B}\end{array}$ & $\begin{array}{l}\text { Bathophenanthrolin o. Enteiweißung, Merck } \\
\text { Roche Wert }\end{array}$ & $\begin{array}{l}590-680-770 \\
630-690-750\end{array}$ & 810 \\
\hline $\begin{array}{l}\text { Moni-Trol II } \\
33 \text { A,B }\end{array}$ & $\begin{array}{l}\text { Bathophenanthrolin o. Enteiweißung, Merck } \\
\text { Roche Wert }\end{array}$ & $\begin{array}{l}1590-1740-1890 \\
1560-1700-1840\end{array}$ & 1930 \\
\hline $\begin{array}{l}\text { Lab-Trol } \\
42 \mathrm{~A}-\mathrm{Z}\end{array}$ & $\begin{array}{l}\text { Bathophenanthrolin o. Enteiweißung, Merck } \\
\text { Bathophenanthrolin o. Enteiweißung, Merck } \\
\text { Roche Wert }\end{array}$ & $\begin{array}{r}1050-1150-1250 \\
1010-1090-1170 \\
950-1040-1130\end{array}$ & 1270 \\
\hline $\begin{array}{l}\text { Patho-Trol } \\
66 \mathrm{~A}-\mathrm{Z}\end{array}$ & Bathophenanthrolin o. Enteiweißung, Merck & $1200-1340-1480$ & 1440 \\
\hline $\begin{array}{l}\text { Roche ,human" } \\
\text { K } 1631\end{array}$ & $\begin{array}{l}\text { Roche Diagnostica-Test } \\
\text { Photometrie (Bathophenanthrolin) } \\
\text { Auto-Analyzer (Tripyridyltriazin) }\end{array}$ & $\begin{array}{l}820-890-960 \\
840-920-1000 \\
900-970-1040\end{array}$ & 990 \\
\hline Roche EZK 50 & Roche Diagnostica-Test & $1520-1650-1780$ & 1730 \\
\hline $\begin{array}{l}\text { Seronorm } \\
120\end{array}$ & 5 versch. Methoden & $1260-1310-1380$ & 1280 \\
\hline
\end{tabular}

Tag. Die Präzision im Lauf lag bei 4,1\% $(n=27)$, Bereich 2,6-5,6\%. Die Präzision von Lauf zu Lauf $(n=10$ Läufe zu je 27 Proben) des Mittelwertes war bei 20,2 $\mu \mathrm{mol} / 1$ $(1126 \mu \mathrm{g} / 1) 7,8 \%$, bei 2 wahllos herausgegriffenen Küvetten über 10 Läufe 7,1 bzw. 8,9\%. Die Präzision von Tag zu Tag ( $\mathrm{n}=10$ Tage) war bei Monitrol I mit $\overline{\mathrm{x}}=14,6 \mu \mathrm{mol} / 18,1 \%$, bei Hyland II mit $\overline{\mathrm{x}}=37,2 \mu \mathrm{mol} / 1$ ebenfalls $8,1 \%$.

\section{Vergleichsuntersuchungen}

Es wurde die Bestimmung der Eisenkonzentration parallel sowohl manuell als auch mit dem CentrifiChem System durchgefuhrt (Abb. 2). Ein relativ großer Teil der untersuchten Seren waren schwach bis mittelmäßig lipämisch bzw. trüb.

Als Reaktionszeit beim CentrifiChem System wurde einheitlich 10 min gewählt, bei einigen Seren wäre eine größere Reaktionszeit bis zur Aufklärung durch Teepol notwendig gewesen. Wie die Versuchsergebnisse zeigten, bedingt die serielle Durchfuihrung der Analysen (,,parallel processing ") bei Zentrifugalanalysatoren für die Bestimmung des Eisens ein spezielles Vorgehen: Entweder werden von der Analyse die lipämischen und trüben Seren ausgesondert und dann in einer gesonderten Serie mit einer Reaktionszeit von $15 \mathrm{~min}$ bestimmt oder es ist notwendig, prinzipiell eine Reaktionszeit von $15 \mathrm{~min}$ zu wählen (ein einziges Serum mit einer speziellen Reaktionszeit determiniert diese Reaktionszeit für die ganze Serie). Als Vergleichsmethode wurde die manuelle Technik gemäß Roche Diagnostica Testpackung (jeweils

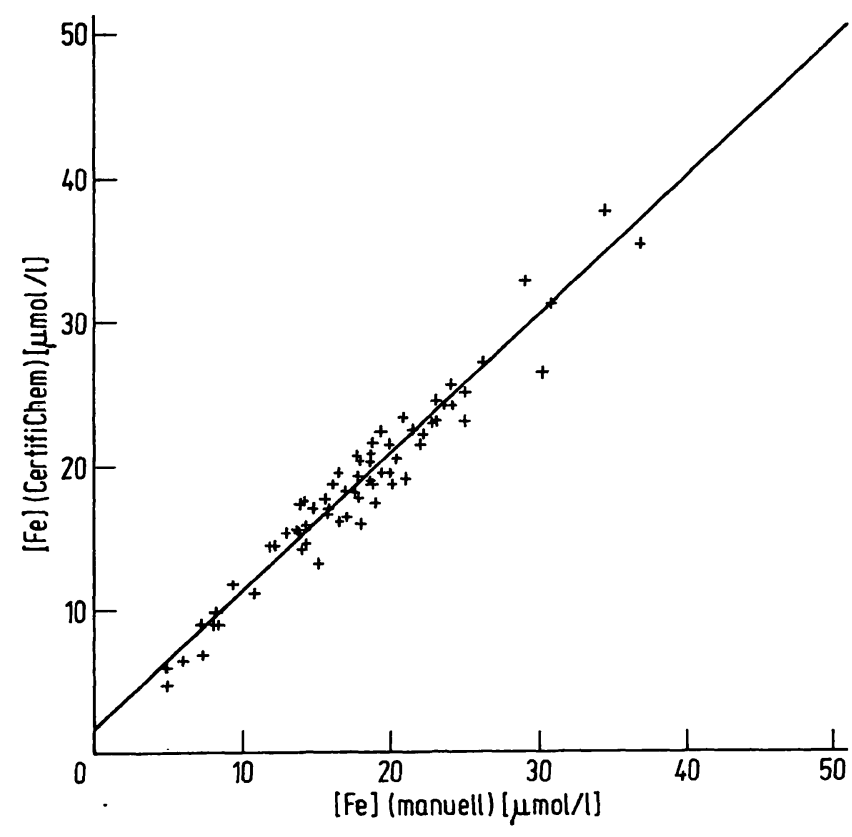

Abb. 2. Vergleich der erhaltenen Meßwerte mit manueller Roche Diagnostica Testpackung und CentrifiChem-Methode.

Einzelversuche) verwendet. Die Summe aller manuellen Werte war 1346,6, die aller CentrifiChem-Werte 1410,6; Mittelwert der manuellen Werte 17,7, der CentrifiChemWerte 18,6 $\mu \mathrm{mol} / 1$. Gleichung der Regressionsgeraden: $y=1,58+0,96 x$, der Korrelationskoeffizient betrug 0,976 . Abbildung 2 zeigt einen Vergleich der erhaltenen Resultate bei der CentrifiChem- und der manuellen Methode. Bei ganz klaren (ausgesuchten) Seren wurde ein Korrelationskoeffizient von 0,990 erhalten. 


\section{Likentur}

1. Trinder, P.. J. Oin. Pathot. $9.170 \cdot 172(1956)$.

2 Shack. A. I., (h ome. J., Rrintiart. R. W.. Milke, ). R., Prix. Sur. Bep. Brid. Mrd. A ? 443 44 (1954).

3. Sihdi. A. Analy th al Appixatkons of 1.10-Phenanthroline and Rolated Componunda Prigamon. (Xxlord 1969.

4. Louber, K., diew 2. $3.96 .99(1963)$.

S. Bambech. M. N.. Klin. Wochenechr. 44. $1276 \sim 1284$ (1966).

6. Sanford, R., J. Clin. Pathol. 16, 174-177 (1963).

D. H. G. Enenwiener

Lat d Abr. Diagnostica

Furmo Hoffmann-La Roche \& Ca, AC

CH-4133 Schweuerhalie 